\title{
Ciliary kinesins beyond IFT: cilium length, disassembly, cargo transport and signaling
}

Madeline Louise Reilly ${ }^{1,2}$ and Alexandre Benmerah ${ }^{1, *}$

${ }^{1}$ Laboratory of Hereditary Kidney Diseases, INSERM UMR 1163, Paris Descartes University, Imagine Institute, 75015 Paris, France.

${ }^{2}$ Paris Diderot University, 75013 Paris, France.

* To whom correspondence should be addressed:

Alexandre Benmerah, Institut Imagine, 24 boulevard du Montparnasse, 75015 PARIS, France.

tel: +33 1427543 44, fax: +33 1427542 25, email: alexandre.benmerah@inserm.fr 


\section{Ciliary Kinesins Beyond IFT}

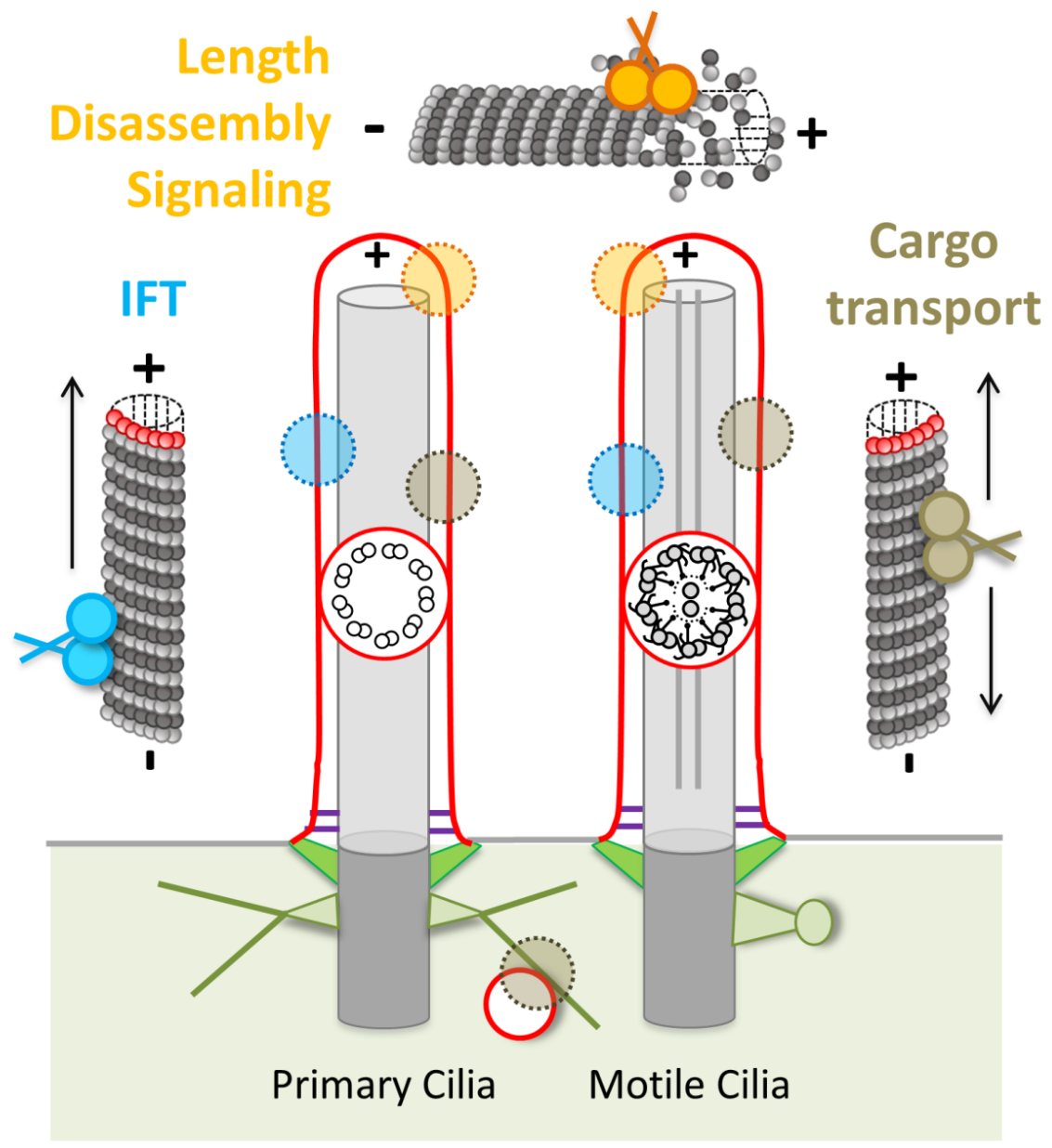

Cilia are highly conserved, microtubule-based organelles subdivided into motile and nonmotile/primary cilia, both playing key roles during development and tissue homeostasis. This review summarizes the ciliary functions of the Kinesin superfamily (KIFs) of microtubule-based motors. Initially implicated in ciliary assembly, KIFs were later shown to be involved in many other key ciliary functions, including cargo transport, signaling (Hedgehog) and the regulation of cilia length and disassembly. 


\begin{abstract}
:
Cilia and flagella are microtubule-based antenna which are highly conserved among eukaryotes. In vertebrates, primary and motile cilia have evolved to exert several key functions during development and tissue homeostasis. Ciliary dysfunction in humans causes a highly heterogeneous group of diseases called ciliopathies, a class of genetic multisystemic disorders primarily affecting kidney, skeleton, retina, lung and the central nervous system. Among key ciliary proteins, kinesin family members (KIF) are microtubule-interacting proteins involved in many diverse cellular functions, including transport of cargo (organelles, proteins, lipids) along microtubules and regulating the dynamics of cytoplasmic and spindle microtubules through their depolymerizing activity. Many KIFs are also involved in diverse ciliary functions including assembly/disassembly, motility and signaling. We here review these ciliary kinesins in vertebrates and focus on their involvement in ciliopathyrelated disorders.
\end{abstract}




\section{Abbreviations}

BB: basal body

BBS: Bardet Biedl Syndrome

CSF: cerebrospinal fluid

IFT: intraflagellar transport

KIF: Kinesin family member

PC: primary cilia

PCD: Primary ciliary dyskinesia

SHh: Sonic Hedgehog 


\section{Kinesins: microtubule based motors and beyond}

Kinesin superfamily (KIF) members are highly conserved proteins characterized by the presence of a motor domain which binds microtubules in an ATP-dependent manner and can be involved in various functions. The motor domain is present at either the $\mathrm{N}$ - or $\mathrm{C}$-terminus or in the middle of the protein sequence (Figure 1), defining three structurally and functionally different kinesin families ( $\mathrm{N}-$, $\mathrm{C}$ - and M-KIFs). Motor domains are usually followed (in the case of N- and M- KIFs) or preceded (in C-KIFs) by both coiled-coil regions, allowing homo- or heterodimerisation, and diverse interacting motifs, promoting binding to very different sets of cargo (microtubules, proteins, lipids). Some Kinesins also interact with accessory, or light chains, which can be involved in cargo binding and or activation of the motor domain (Hirokawa et al., 2009). Kinesins are frequently maintained in an inactive state and require either cargo or light/accessory chain binding or phosphorylation events to release the constitutive inhibition of the motor domain (Verhey and Hammond, 2009).

In the classical motor Kinesins (Kinesins-1/2/3), with an N-terminal motor domain, ATP hydrolysis allows the movement of the kinesin toward the growing plus $(+)$ ends of microtubules in a "walking" like process. C-Kinesins (Kinesin-14) function similarly except that they move towards the minus-end of microtubules, reminiscent of dyneins. Finally, M-Kinesins (Kinesin-13) are not motile and possess microtubule depolymerizing activity, again mediated by the motor domain (Hirokawa et al., 2009). However, there are exceptions to this general classification, such as KIF7 (Kinesin-4), an immotile N-KIF which binds microtubule + ends directly and promotes their disassembly (He et al., 2014; Yue et al., 2018). Additionally, KIF19A (Kinesin-8) presents both plus-end directed motility and depolymerizing activity, both of which are mediated by the motor domain (Niwa et al., 2012; Wang et al., 2016).

These differing functions allow Kinesins to play key roles in various microtubule-dependent processes, including vesicular trafficking and organelle transport (Hirokawa et al., 2009), as well as the organization and dynamics of the mitotic spindle (Cross and McAinsh, 2014). A number of Kinesin family members also have cilia-specific activities. In addition to the well-established role of Kinesin-2 in ciliogenesis (Scholey, 2013), recent works have highlighted the role of many other KIFs in diverse cilia-related functions, including vesicular trafficking, control of cilia length and ciliary disassembly.

\section{Biogenesis of Primary and Motile cilia.}

Cilia and flagella are highly conserved cellular structures present in most eukaryotes. They are all assembled from basal bodies (BB), which in vertebrates correspond to the mother centriole of the centrosome, in the case of primary cilia, and to amplified centrioles or BBs, in the case of the motile cilia of multiciliated cells. During ciliogenesis (Figure 2), centrioles dock onto cellular membranes 
through their distal appendages (transition fibers) and their $A / B$ tubules extend to form the axoneme, the shared architecture/skeleton of all cilia, composed of 9 peripheral doublets of microtubules. A major structural difference observed between cilia, and frequently associated with the number of cilia present per cell, is the presence of an additional central pair of microtubules. This structural difference, often associated with motility, is easy to observe in transverse sections following electron microscopy. Cilia have subsequently been classified as ' $9+0$ ' and ' $9+2$ ' based upon the absence or presence, respectively, of the central pair of microtubules (Satir and Christensen, 2007; Ishikawa and Marshall, 2011).

In vertebrates, or at least in mammalian cells, ciliogenesis occurs primarily in quiescent or post-mitotic terminally differentiated cells. The initial phase of this highly conserved process is the anchoring of the BB to cellular membranes through their distal appendages, which are then called the transition fibers. In most cases, the first membranes onto which BB dock are intracellular vesicles (Sorokin, 1968; Park et al., 2008; Wu et al., 2018). These incoming vesicles (primary ciliary vesicles) will fuse to form the ciliary vesicle and cap the distal part of the BB (Bernabé-Rubio and Alonso, 2017). This step will lead to the removal of CP110, a key regulator of ciliogenesis, allowing the elongation of the axoneme (Sánchez and Dynlacht, 2016). Following the formation of the transition zone, ciliary proteins are actively transported into the ciliary compartment through a highly conserved process, intraflagellar transport (IFT), initially identified in Chlamydomonas reinharditi. During this complex transport, ciliary components (cargo) are captured in the cytoplasmic compartment by IFT complexes which dock onto transition fibers, cross the transition zone and move along doublets of microtubules of the forming axoneme. The subsequent plus-end directed, or anterograde, transport requires Kinesin-2 motor proteins (Scholey, 2013). The BBSome, a complex formed by products of Bardet-Biedl Syndrome (BBS) genes (see below), also participates in the transport of ciliary membrane proteins through interactions with IFT trains. After remodeling at the distal tip of cilia and the dissociation of anterograde cargo, IFT/BBS complexes undergo retrograde IFT transport, requiring cytoplasmic dynein 2 which mediates the recycling of IFT/BBS complexes in the cytoplasmic compartment (Lechtreck, 2015; Nakayama and Katoh, 2018) and the exit of specific cargo (GPCRs, signaling proteins) from the ciliary compartment (Nachury, 2018). These processes are highly conserved among eukaryotes and are responsible for the biogenesis of the great majority of cilia. In addition to IFT and BBSome, the CPLANE complex, which shares some elements with components of the PCP pathway, was recently identified in vertebrates as a regulator of retrograde IFT components required for ciliogenesis (Adler and Wallingford, 2017).

Ciliogenesis is governed by the expression of specific 'ciliary' genes (IFT, BBS) which are controlled by transcription factors from the RFX family, as well as FOXJ1, a master regulator of motile cilia biogenesis (Thomas et al., 2010; Meunier and Azimzadeh, 2016). In addition to the basic 
ciliogenesis machinery, an additional set of genes must be activated in the case of centriole biogenesis to allow amplification of centrioles (deuterosome) and the formation of axonemal structures required for motility (dynein arms, radial spokes, central pair).

Ciliary disassembly in vertebrates mainly concerns primary cilia (see below), which can be formed in cells retaining the potential to proliferate (stem cells, neuron progenitors and fibroblasts). When ciliated $\mathrm{G}_{0}$ cells are stimulated, through serum addition in vitro, and re-enter the cell cycle, specific sets of proteins are activated. The activation of these proteins, including kinesin family members, will lead to the depolymerization of axonemal microtubules. This step is required to allow the duplicating centrosome to detach from the plasma membrane, which is then free to participate in the assembly of the mitotic spindle (Sánchez and Dynlacht, 2016).

\section{Cilia/ciliopathies subtypes:}

Primary cilia (PC) are sensory cilia with key roles during development in vertebrates. They were called 'primary' by Sorokin as they are the first to appear during development, even in cells which will assemble multiple motile cilia (Sorokin, 1968). They are characterized by a ' $9+0$ ' axoneme and a BB composed of two centrioles, corresponding to the unique centrosome of the cell. They can be divided in two groups depending upon the presence of a ciliary pocket at their base (Ghossoub et al., 2011). However, the specific functional consequences associated with the presence or absence of the ciliary pocket remains to be fully determined (Pedersen et al., 2016).

Sensory cilia were first characterized in photoreceptors. The photoreceptor outer segment corresponds to a modified cilium which is connected to the inner segment through the connecting cilium (DE ROBERTIS, 1956). Based on these observations, it was suggested that similarly organized cilia found in other tissues may play sensory or signaling functions (BARNES, 1961). Support for these suggested roles came from the establishment of the link between dysfunctions of ' $9+0$ ' cilia and complex human genetic diseases, subsequently termed 'ciliopathies' (Badano et al., 2006b). Studies in the last 25 years have since provided strong evidence that PC control numerous key signaling pathways during development and tissue homeostasis (Wheway et al., 2018). In addition to the sensation of urine flow in renal epithelial cells, the archetype of these cilia-dependent pathways is Sonic Hedgehog (SHh). While most tissues in Drosophila are unciliated and thus $\mathrm{Hh}$ signaling is independent of cilia, this is not the case in vertebrates. Here, the complex in and out trafficking events of SHh signaling components control the downstream activation or downregulation of the pathway. This control is effected by the activation or generation of repressor forms of the GLI2 and GLI3 transcription factors (Bangs and Anderson, 2017).

Ciliopathies, linked to dysfunctions of primary/sensory cilia, were initially identified in the context of polycystic kidney disease (Barr and Sternberg, 1999; Pazour et al., 2000). Cystic kidney 
was, therefore, one of the first manifestations identified as a cilia-related phenotype. Many phenotypes have subsequently been associated with PC ciliopathies (see below) and the broad spectrum of affected organs reflects the presence of a PC in almost all vertebrate cell types and their key functions during development and/or tissue homeostasis. The notion of a "ciliopathic" disorder was first attributed to Bardet-Biedl syndrome (MIM \#209900, BBS), a condition characterized by retinal dystrophy, cystic kidneys, polydactyly and obesity. Central nervous system defects are also observed in ciliopathies highlighting the important role of cilia during brain development (GuemezGamboa et al., 2014; Thomas, Reilly and Benmerah, manuscript in preparation). Most if not all the genes mutated in these PC-Ciliopathies encode ciliary proteins, which can be classified in functional subdomains. Meckel and Joubert syndromes are largely caused by mutations in genes encoding transition zone proteins, while BBS results from mutations in genes encoding BBSome components, and skeletal ciliopathies from mutations in genes encoding retrograde IFT and cytoplasmic dynein 2 subunits (Huber and Cormier-Daire, 2012; Braun and Hildebrandt, 2017; Reiter and Leroux, 2017).

Motile cilia are present at the apical surface of epithelial cells in several tissues, including the upper airways (bronchus, nasal epithelium), fallopian tubes and lateral ventricles (ependymal cells). Their coordinated beating is based on planar cell polarity signaling and is required for the movement of fluids present at the apical surface of these multiciliated cells (Meunier and Azimzadeh, 2016). Motile cilia are usually characterized by a ' $9+2$ ' axoneme and the presence of additional structures required for motility, including dynein arms (inner and outer), radial spokes and nexin links, as well as by intradoublet structures such as tektin filaments (Figure 2). There are, however, non-motile ' $9+2$ ' cilia (olfactory neurons, hair cells) which lack accessory structures, and motile ' $9+0$ ' cilia (the node in mammals, Kupffer's vesicle in zebrafish) which present dynein arms. When involved in the motility of the cell upon which they are present, motile cilia are called flagella (sperm cells). Flagella of sperm cells present a modified BB along with additional structures along the axoneme involved in motility (Satir and Christensen, 2007).

Primary ciliary dyskinesia $(P C D)$ is linked to motile cilia dysfunction, including perturbed motility and/or a reduction in the number of motile cilia at the surface of ciliated cells. PCD comprises chronic airway diseases, otitis media and sinusitis, all of which are linked to motile cilia defects in the airways, and male infertility. In Kartagener syndrome, these manifestations are associated with situs inversus, which is caused by defects of node cilia and can lead to congenital heart disease. PCD results from mutations in genes encoding proteins required for motile cilia function or biogenesis, including dynein arms, radial spoke components and transcription factors, as well as cytoplasmic chaperones required for the assembly of dynein arms (Mitchison and Valente, 2017; Reiter and Leroux, 2017). 
The identification of the cellular machineries required for ciliary assembly and regulation were made in several models, including cultured mammalian cell lines and two key model organisms, the biflagellated green algae Chlamydomonas reinhardtii and the worm Caenorhabditis elegans. Many of the actors involved in motile and sensory cilia assembly and regulation were initially described in these two organisms, respectively. In addition, both mouse and zebrafish have been used to generate models of ciliopathies and to further characterize the role of cilia subtypes and ciliary proteins in vertebrates (Vincensini et al., 2011). Although the phenotypes in mouse models are frequently reminiscent of those found in human ciliopathies, the use of zebrafish larvae has been crucial to characterize the function of a number of ciliary genes (Song et al., 2016). In addition to various experimental advantages, zebrafish have recently revealed the role of motile cilia in development of the spine, implicating, therefore, motile cilia dysfunction in scoliosis (Grimes et al., 2016). Additionally, a ventrally curved body axis, known to be a ciliopathy-associated phenotype in zebrafish but for which the etiology was not clearly understood, has recently been linked to the crucial role of motile cilia in the organization of the Reissner fibre. The Reissner fibre is a component of the cerebrospinal fluid in vertebrates, including mammals, and is essential for the morphogenesis of a straight posterior body axis (Cantaut-Belarif et al., 2018).

The key role of Kinesins at cilia was first identified through the observation of the requirement of Kinesin 2 family members for IFT in Chlamydomonas (Kozminski et al., 1995). Additional Kinesin family members were subsequently shown to be involved in regulating cilia length, ciliary disassembly and to have motile cilia-specific functions. Here, we have focused on studies performed using vertebrates and mammals in relation to human ciliopathies and diseases (Table 1).

\section{IFT transport:}

The role of Kinesin-2 in anterograde IFT was comprehensively reviewed elsewhere (Scholey, 2013; Lechtreck, 2015) and will be described very briefly here. While both PC and motile cilia assembly require functional IFT, and therefore the KIF3A/B anterograde motor, the role of KIF17 in vertebrates remains to be clearly determined.

\section{KIF3A/KIF3B/KAP3 (Kinesin-2):}

Heterotrimeric Kinesin-2, composed of homo- or heterodimers of KIF3A and KIF3B associated with the accessory subunit KAP3, mediates anterograde IFT through interactions with IFT-B subunits (Scholey, 2013; Nakayama and Katoh, 2018). Initially shown in Chlamydomonas, the essential role of kinesin-2 in anterograde IFT and ciliogenesis was also later demonstrated in many different 
organisms, including mouse and zebrafish. KIF3C, which is able to dimerize with both KIF3A and $\mathrm{KIF} 3 \mathrm{~B}$, is not required for ciliogenesis. Members of this subfamily have also been implicated in vesicular trafficking (Hirokawa et al., 2010; Scholey, 2013). However, despite contributing to the transport of post-Golgi vesicles and endosomes, the potential role of Kinesin-2 in the trafficking of the primary ciliary vesicles has not yet been investigated.

\section{KIF17 (kinesin-2):}

Kif17 functions as a homodimer and was shown to mediate anterograde IFT along the distal singlets of microtubules within the sensory cilia of the worm, and to be required for the assembly of this singlet only region (Snow et al., 2004). However, the ciliary function of KIF17 in vertebrates remains puzzling (Scholey, 2013). While the morpholino-mediated KD of kif17 in zebrafish was shown to lead to retinal degeneration (Insinna et al., 2008), the invalidation of KIF17 in both zebrafish (Zhao et al., 2012) and mouse (Yin et al., 2011; Jiang et al., 2015) did not lead to any significant ciliary phenotypes besides a small delay in outer segment development in Zebrafish (Lewis et al., 2017). Recent works also indicate that the activity of KIF17 controls outer segment turnover (Lewis et al., 2018). Although singlet microtubule regions were identified in the distal segments of sensory cilia in mammals, including olfactory neurons and in the outer segment of photoreceptors (Falk et al., 2015), the singlet specific transport function of KIF17 does not appear to be conserved in vertebrates. KIF17, along with heterodimeric kinesin-2, has been shown to participate in IFT along the entire axoneme of olfactory cilia (Williams et al., 2014). KIF17 was additionally implicated in the transport to the cilium of several membrane proteins with important functions in signaling (Jenkins et al., 2006; Leaf and Von Zastrow, 2015). Kif17, along with KIF3A/3B, plays a well-documented role in vesicular transport in the dendrites of neurons, which has been shown to be involved in memory (Yin et al., 2011; Scholey, 2013). Again, a link between these vesicular transport-related functions and its roles at cilia is still missing.

\section{PC-specific Kinesins}

\section{Axonemal microtubule dynamics:}

In addition to their role in IFT, several KIFs have more recently been shown to function in regulating the length of the axoneme or in the disassembly of the axoneme during cell cycle re-entry. While such roles could be expected for members of the Kinesin-13 subfamily, known to have microtubule depolymerizing activities, these functions were only demonstrated more recently for the Kinesin-4 subfamily. Described below are KIFs which appear to play a role at PC. In addition to these PC-specific depolymerizing KIFs, another set of KIFs appear to regulate the length of motile cilia and are described thereafter. 
KIF7 (Kinesin-4):

The ciliary and SHh-related roles of KIF7 have recently been reviewed elsewhere (Bangs and Anderson, 2017). Briefly, KIF7 is a one of the two Costal2 orthologues in mammals (with KIF27, see below) and is a key actor of PC-dependent SHh signaling in vertebrates (Cheung et al., 2009; EndohYamagami et al., 2009; Liem et al., 2009; Wilson et al., 2009). Mutations in KIF7 were identified in ciliopathies characterized by developmental brain anomalies linked to impaired SHh signaling (Dafinger et al., 2011; Putoux et al., 2011, 2012). Initially thought to be involved in the intraciliary transport of SHh signaling components (GLI2, SuFu), KIF7 was recently shown to lack motor activity. Instead, KIF7 possesses microtubule depolymerizing activity and, therefore, negatively controls the length of axonemal microtubules in order to organize a SHh signaling platform at the homogenouslysized distal ends of ciliary microtubules (He et al., 2014).

\section{KIF2A (Kinesin-13):}

KIF2A is the prototype of the Kinesin-13B subfamily which plays various roles in mitotic spindle dynamics/organization (Walczak et al., 2013). KIF2A was shown to be involved in the disassembly of cilia upon cell cycle entry through a PLK1-mediated activation of its depolymerizing activity (Miyamoto et al., 2015). Loss of Kif2A in mice leads to severe brain defects and dominant KIF2A mutations in humans lead to microcephaly (Poirier et al., 2013), due, at least partially, to the cell cycle delay in neuron progenitors resulting from cilia disassembly defects (Broix et al., 2018). Intriguingly, besides brain anomalies, loss of Kif2A in mouse does not lead to obvious ciliopathy phenotypes, suggesting that its role in ciliary disassembly might be brain specific. It may also reflect a specific requirement for ciliary disassembly in neuron progenitors which are among the very rare ciliated cells which are also highly proliferative, requiring fast ciliary assembly/disassembly cycles (Thomas, Reilly and Benmerah, manuscript in preparation).

\section{KIF24 (Kinesin-13):}

KIF24 is the unique member of Kinesin-13A subfamily. It is a centriolar protein which was initially identified as a partner of CP110. KIF24 is required for the recruitment/stabilization of CP110 at the distal end of the mother centriole and therefore negatively regulates the early steps of ciliogenesis (Kobayashi et al., 2011). KIF24 was also shown to possess microtubule depolymerizing activity, specifically targeting centriolar microtubules (Kobayashi et al., 2011). This depolymerizing activity is in fact triggered during cell cycle reentry via phosphorylation by NEK2 and contributes to cilia disassembly (Kim et al., 2015). Of note and surprisingly, KIF24 is not conserved in zebrafish. The effects of the in vivo invalidation of KIF24 have not yet been investigated. Interestingly, loss of CP110 
in mouse causes severe developmental defects reminiscent of skeletal ciliopathies (Yadav et al., 2016), making both CP110 and KIF24 good candidate genes for skeletal ciliopathies in humans. Finally, KIF24 presents a SAM domain in the N-terminal region. The role of this domain within KIF24 has not yet been investigated but could potentially establish functional links with other SAM domaincontaining proteins involved in ciliopathy-related disorders, including BICC1, ANKS6 and ANKS3 (Kraus et al., 2012; Hoff et al., 2013; Shamseldin et al., 2016).

\section{Recently identified PC-specific kinesins.}

In addition to IFT and depolymerizing kinesins involved in PC assembly/disassembly cycles, recent research has identified several new Kinesin family members involved in diverse functions at PC.

\section{KIF5B (Kinesin-1):}

Kinesin-1 family members are the prototypes of dimeric motor kinesins and have been shown to be responsible for the movement of many different cargos (endosomes, lysosomes, nuclei) along cytoplasmic microtubules (Hirokawa et al., 2010). While KIF5B is ubiquitously expressed, KIF5A and KIF5C are brain specific (see below). The role of KIF5B at cilia was identified based on an interaction with the product of MGC1203/CCDC28 (Novas et al., 2018), a modifier of BBS (Badano et al., 2006a). CCDC28 interacts with BBSome subunits (Badano et al., 2006a) and with SIN1 (Cardenas-Rodriguez et al., 2013), an mTORC2 subunit, to positively regulate cilium length. KIF5B and its associated light chain (KLC1) appear to negatively regulate CCDC28, and, therefore, their downregulation result in longer cilia. KIF5 and KLC1 were both shown to localize to the BB regions and could not be detected in the cilium (Novas et al., 2018). It is currently unclear whether they present an intraciliary IFT motor-like role, or if they are involved in the cytoplasmic transport of CCDC28B and partners. Invalidation of KIF5B in the mouse is embryonic lethal, although whether the mutant embryos presented with ciliopathy-related phenotypes was not described (Tanaka et al., 1998). In Zebrafish, KIF5B isoforms were involved in cartilage remodeling and maintenance during craniofacial morphogenesis, but without an obvious connection to cilia-related function (Santos-Ledo et al., 2017). Loss of KLC1 in the mouse leads to brain specific defects which were not related to cilia (Rahman et al., 1999).

\section{KIFC1/HSET (Kinesin-14):}

KIFC1 is a member of the Kinesin-14 family and was shown to play an important role during mitotic spindle assembly through microtubule bundling and sliding activities (Cross and McAinsh, 2014). A recent study has shown that KIFC1 is required for the early steps of ciliogenesis, before the removal of CP110 from the mother centriole (Lee et al., 2018). In addition, this study also showed that KIFC1 
interacts with ASAP1, implicated in the transport of membrane proteins from the Golgi apparatus to the outer segment in photoreceptors (Mazelova et al., 2009). Taken together these data indicate that KIFC1 may participate in the delivery of ciliary vesicles to the mother centriole during the early steps of ciliogenesis and suggest a general role in Golgi to PC trafficking. KIFC2 and KIFC3, which were previously implicated in vesicular transport and Golgi organization (Hirokawa et al., 2010), do not seem to play similar ciliary functions (Lee et al., 2018). Interestingly, KIFC1 was also involved in the transport of endosomes through an interaction with KIF5B (Nath et al., 2007) suggesting that they might functionally interact during ciliogenesis. To our knowledge, there is currently no published study on the impact of the invalidation of KIFC1 on cilia in vivo, however invalidation in the mouse does not appear to lead to an obvious phenotype (Hirokawa et al., 2010). Similarly, no phenotype is evident upon invalidation of KIFC2 or KIFC3 (Yang et al., 2001b, 2001a; Xu et al., 2002), suggesting a possible compensation between Kinesin-14 family members in vivo.

\section{KIF13B/GAKIN (kinesin-3):}

KIF13B/Gakin is a member of the large Kinesin-3 family which was previously involved in endosomal transport (Hirokawa et al., 2010) as well as in caveolae-mediated trafficking (Kanai et al., 2014). A role at PC was identified based on the high expression in serum-starved fibroblasts and was recently extensively reviewed elsewhere (Morthorst et al., 2018). Briefly, KIF13B localizes to the BB and axoneme and has been shown to interact with NPHP4, a key component of the transition zone. KIF13B organizes a caveolin-1 domain at the base of cilia which appears to be required for efficient ciliary trafficking of smoothened and downstream SHh signaling (Schou et al., 2017). Invalidation of Kif13b in the mouse does not lead to an obvious phenotype (Kanai et al., 2014). Interestingly, KIF13B is close to the mammalian orthologue of KIp-6, which was identified in the worm as a cilia subtypespecific kinesin involved in IFT regulation and targeting of PKD2 to cilia and extra-cellular vesicles (Peden and Barr, 2005; Wang et al., 2014). The rat KLP6 was involved in the transport of mitochondria (Tanaka et al., 2011), although a putative ciliary function was not investigated. The closest orthologue of Klp-6 in human, KIF28, is considered a pseudo gene.

\section{Potential additional PC-specific KIFs?}

\section{KIF11/EG5 (Kinesin-5):}

$\mathrm{KIF11/Eg5} \mathrm{is} \mathrm{a} \mathrm{key} \mathrm{mitotic} \mathrm{kinesin} \mathrm{required} \mathrm{for} \mathrm{the} \mathrm{assembly} \mathrm{of} \mathrm{a} \mathrm{bipolar} \mathrm{mitotic} \mathrm{spindle} \mathrm{(Sawin} \mathrm{et} \mathrm{al.,}$ 1992). The crucial role of KIF11/Eg5 during development was first identified in Drosophila where the loss of KLP61F results in strong defects in proliferative tissues, including in the larval brain (Heck et al., 1993). Invalidation of Kif11 in the mouse results in an early block in embryogenesis, prior to the 
implantation stage (Castillo and Justice, 2007; Chauvière et al., 2008), whereas in zebrafish, it leads to a severe delay in brain development (Amsterdam et al., 2004) linked to the mitotic block (monopolar spindle) and apoptosis of progenitors (Johnson et al., 2014). The latter results in zebrafish suggest a shared and important role during brain development in vertebrates. In humans, autosomal dominant mutations in KIF11 were identified in individuals presenting with microcephaly with or without chorioretinopathy, lymphoedema, or mental retardation (MCLMR; MIM \#152950; (Ostergaard et al., 2012)). Despite recent evidence for non-mitotic functions related to vesicular trafficking (Wakana et al., 2013), there was no strong evidence for a potential role of KIF11 at cilia and its key role in spindle assembly was sufficient to explain the delay in the proliferation of neuron progenitors and subsequent microcephaly. However, it was recently shown that the spectrum of KIF11-associated retinopathy includes a new form of retinal ciliopathy and that KIF11 localizes to the connecting cilium of photoreceptors suggesting a potential function at sensory/primary cilia and that KIF11-related diseases represent syndromic ciliopathies (Birtel et al., 2017). The potential ciliary function for KIF11 in neuron progenitors and its involvement in the KIF11-associated microcephaly remains an open question.

\section{KIF14 (Kinesin-3):}

KIF14 is a member of the kinesin-3 subfamily and was classified as an N-Kinesin despite the presence of an N-terminal extension which binds PRC1 (Gruneberg et al., 2006), a key component of the central spindle. KIF14 is expressed in mitotic cells and is required for cytokinesis (Carleton et al., 2006; Gruneberg et al., 2006). Invalidation of KIF14 in rodents leads to severe microcephaly and growth retardation but no ciliopathy-like phenotypes (Fujikura et al., 2013). In humans, biallelic recessive mutations in KIF14 (Moawia et al., 2017; Makrythanasis et al., 2018) were recently identified in individuals with mild to very severe microcephaly. The pathogenic effects of the mutations were thought to be linked to the major role of KIF14 at the midbody and to impaired cytokinesis. However and interestingly, loss of function mutations in KIF14 in humans cause a syndromic microcephaly associated with cystic renal hypodysplasia (Filges et al., 2013; Heidet et al., 2017). In addition, as the presence of cystic kidneys and developmental brain defects in the affected foetuses were reminiscent of Meckel-Grüber syndrome, KIF14 was therefore termed MKS12. These observations suggested a potential link between KIF14 and PC, however, our recent works failed to establish such a functional link (Reilly et al., 2018).

\section{Motile cilia Kinesins}

In addition to the shared function of Kinesin-2 family members in the IFT-dependent formation of cilia, other Kinesins appear to play specific functions in motile cilia. Interestingly, the roles of most of 
these KIFs in motile cilia in vertebrates were identified or confirmed in screens aiming to identify targets of FOXJ1.

\section{KIF27 (Kinesin-4):}

KIF27 is a mammalian-specific paralogue of KIF7, the two mammalian orthologues of Costal2 (Wilson et al., 2009). KIF27 was, therefore, expected to play a major role in SHh signaling in vertebrates, resembling KIF7 (see above). Surprisingly, KIF27 is not conserved in zebrafish (Wilson et al., 2009), and invalidation in the mouse did not lead to the expected SHh phenotypes (Chen et al., 2005), indicating that it is unlikely to play a conserved role in this pathway. Interestingly, the unique Costal2 orthologue in planarians is not involved in SHh signaling, but is required for ciliogenesis of motile cilia (Rink et al., 2009). Similarly, loss of Kif27 in the mouse leads to PCD-like phenotypes, including sinusitis, otitis media and hydrocephalus (Vogel et al., 2012), suggesting a motile cilia-specific role in mammals. KIF27 localizes to BB in tracheal epithelial cells (Wilson et al., 2009) and oviduct epithelial cells (Nozawa et al., 2013), but a precise function in motile cilia was not further investigated. Interestingly, and reminiscent of Costal2 in Drosophila, KIF27 interacts with Fused/Stk36, which, again, is not involved in SHh signaling in vertebrates. Fused is required for the assembly of the central pair of microtubules in motile cilia (Wilson et al., 2009) and KIF27 may participate in this Fusedmediated function. In vitro studies have shown that KIF27 presents slow processivity (Yue et al., 2018), although the dynamics within motile cilia and the in vivo consequences of this processive activity remain to be investigated. Intriguingly, KIF27 was found at the tip of PC when ectopically expressed in mesenchymal cells (Nozawa et al., 2013), and while the impact of this finding remains to be investigated, this localization is suggestive of a + end directed motion.

\section{KIF9 (Kinesin-9):}

KIF9, and KIF6 (see below), are similar to KLP1 which was identified in Chlamydomonas as a component of the central pair (Bernstein et al., 1994), where it is required for motility, likely through the regulation of dynein activity (Yokoyama et al., 2004). KLP1 also interacts with Hydin (Lechtreck and Witman, 2007) which has been implicated in PCD in humans (Olbrich et al., 2012). The orthologue of KLP1 in trypanosomes, termed KIF9A, localizes along the axoneme and is also required for motility (Demonchy et al., 2009). KIF9 is highly expressed in sperm cells in both mammals (Chauvin et al., 2012) and Ciona intestinalis (Konno et al., 2015). In addition, KIF9 was identified in a transcriptomic analysis during mucociliary differentiation in human airway epithelial cells (Ross et al., 2007) and as a target of FOXJ1 in murine ependymal cells (Jacquet et al., 2009). The ciliary function of KIF9 remains to be investigated in vertebrates, where it has been implicated in the classical 
microtubule-based transport of various cargoes in non-ciliated cells (Cornfine et al., 2011; Gache et al., 2017).

\section{KIF6 (Kinesin-9):}

KIF6 is a paralogue of KIF9, termed KIF9B in trypanosomes, where it localizes to both the BB and axoneme and is involved in the assembly of the paraflagellar rod, an extra-axonemal structure required for motility (Demonchy et al., 2009). KIF6 was identified as a target of FOXJ1 in both mouse (Jacquet et al., 2009) and zebrafish, where it localizes to the BB region of motile cilia (Choksi et al., 2014). However, knockdown of kif6 did not lead to classical cilia-related phenotypes in Zebrafish larvae, aside from a curved body axis (Buchan et al., 2014), which could potentially be indicative of a role in motile cilia in ependymal cells (Grimes et al., 2016) and/or in Reissner fiber biogenesis (Cantaut-Belarif et al., 2018). Interestingly, mutations in kif6 in zebrafish lead to scoliosis (Buchan et al., 2014), which has recently been linked to abnormalities in CSF flow caused by defects in motile cilia in the spine and brain ventricles. The motile cilia-related function of KIF6 was expected to be conserved in mammals based on its expression in ciliated tissues (Buchan et al., 2014). Kif6 invalidated mice did not appear to present typical PCD-like phenotypes (Hameed et al., 2013). However and interestingly, a recent report indicates that KIF6 plays a crucial and specific role in ciliogenesis in ependymal cells and that its loss in both species leads to enlargement of brain ventricles (Konjikusic et al., 2018). It is not currently known whether this role is conserved in humans although this study also characterizes biallelic KIF6 mutations in a case of macroencephaly with intellectual disabilities.

\section{KIF18B (kinesin-8):}

KIF18b is a member of the Kinesin- 8 family and was previously characterized as a negative regulator of the microtubule dynamics of the mitotic spindle (Stout et al., 2011; Tanenbaum et al., 2011), by promoting microtubule catastrophe (McHugh et al., 2018). In parallel, it was identified as a target of foxj1 in Zebrafish. Kif18b is present at the BB region of motile cilia and typical ciliopathy-related phenotypes were observed in morpholino-treated larvae, supporting its key role in motile cilia biogenesis/function in this organism (Choksi et al., 2014). Surprisingly, the loss of kif18b results in shorter cilia in the pronephros (Choksi et al., 2014), which was unexpected given the known function of KIF18B at the spindle in mammals. This result suggests either that the function at ciliary microtubules is different compared to cytoplasmic microtubules or that kif18b acquired a different function in Zebrafish. Interestingly, KIF18B was also shown to interact with EB1 (Stout et al., 2011), a positive regulator of microtubule growth and ciliogenesis (Schrøder et al., 2011), which may provide 
a clue to understanding the ciliary functions of KIF18B. To our knowledge, there is no mouse model for Kif18B and its ciliary function in vivo remains to be investigated in mammals.

\section{KIF19A (kinesin-8):}

KIF19A was an uncharacterized Kinesin-8 whose function was directly investigated via knockout in mouse, which led to strong motile cilia-related phenotypes, including hydrocephalus and female infertility (Niwa et al., 2012). KIF19A possesses a ' + ' end directed motor activity and localizes to the distal end of the axoneme where it negatively controls ciliary length through its microtubule depolymerizing activity (Niwa et al., 2012; Wang et al., 2016). The loss of KIF19A causes elongation of motile cilia in all tissues examined thus far, impaired beating and subsequently inefficient fluid flow (Niwa et al., 2012). A potential role in the regulation of primary cilia length was not investigated, however, the lack of PC-related phenotypes in the invalidated mouse model suggests that the loss of Kif19A does not severely impact upon PC-dependent signaling pathways.

\section{Different cilia, different set of kinesins?}

It appears that a specific set of kinesins were selected during evolution to exert motile cilia-specific functions in vertebrates and particularly in mammals. Indeed, Kinesin-8 family members, including KIF18B which was initially characterized as a mitotic kinesin, play unanticipated roles in cilium length regulation, confirming the current use of mitotic proteins in ciliary functions and vice versa (Hua and Ferland, 2018). Interestingly, it was recently shown that the CDK1 cell cycle signaling axis is partially reactivated during the differentiation of ependymal cells (Al Jord et al., 2017). This result may also explain how some proteins mainly expressed/activated in mitosis, including kinesins, could additionally possess ciliary functions in these cells. Another striking example of the acquisition of motile cilia-specific functions is KIF27, which is specific to mammals and has evolved together with its partner Fused to play a key role at motile cilia. This may highlight the need to tightly couple ciliogenesis and the length of motile cilia at steady-state in different ciliated tissues, where they are required to generate the efficient flow of fluids of different composition/viscosities.

Intriguingly, it seems that different sets of Kinesins were selected to control ciliary length in primary versus motile cilia. While Kif19A invalidated mice do not show evidence of PC-associated phenotypes (Niwa et al., 2012), Kif2A invalidated mice do not show PCD-like phenotype (Homma et al., 2003). Although CP110 plays key roles in both primary and motile cilia ciliogenesis (Song et al., 2014; Walentek et al., 2016), the function of KIF24 has not yet been investigated in the context of motile cilia. Notably, both KIF2A and KIF24 are involved in PC disassembly during cell cycle re-entry, a process which has not been described and is not expected to occur in post-mitotic, multiciliated cells. In contrast, motile cilia disassembly does occur prior to cell division in Chlamydomonas, as well as 
other unicellular eukaryotes, or in response to various stresses, and Kinesin-13 orthologues have been implicated in these processes (Hu et al., 2015). The potential role of KIF7 at motile cilia has not been investigated directly. Interestingly, the loss of kif7 in zebrafish causes situs inversus (Wilson et al., 2009), a phenotype not observed in the context of KIF7 mutations in humans, suggesting a role at motile cilia in the laterality organ of this organism. Additionally, hydrocephalus is often present in individuals harboring KIF7 mutations (Putoux et al., 2011), suggesting a specific role at motile cilia in ependymal cells. However, SHh signaling plays a critical role in ependymal cell specification/differentiation (Yu et al., 2013), possibly indicating that hydrocephalus in the absence of KIF7 can be a consequence of impaired SHh signaling. Finally, KIF7 mutations do not lead to classical PCD-like phenotypes, suggesting that it is unlikely to play a general role at motile cilia.

\section{General conclusion}

Numerous Kinesins appear to play important and specialized roles at primary and/or motile cilia, including assembly through IFT, cell cycle-regulated disassembly, axonemal length control and signaling (SHh). Most kinesins represent good candidate genes for ciliopathies but only a few have so far been reported. An outstanding question remains whether all ciliary KIFs are now known? Redundancy among KIF family members, their involvement in crucial cytoplasmic functions, and/or restricted function in the transport of specific sets of cargos to cilia might have delayed their identification. In addition to the proteomic and transcriptomic data already available, the improvement of proximity labeling approaches and single cell transcriptomic analysis of ciliated tissues may provide new and interesting data in order to identify the expression of specific KIFs in cells where cilia are known to have crucial functions. 


\section{Acknowledgements:}

The work in the lab of the authors was supported by the Fondation pour la Recherche Médicale (DEQ20130326532) and the GIS-Institut des Maladies Rares (AMA11025KSA).

\section{Conflict of interest:}

None to declare. 


\section{References:}

Adler, PN, and Wallingford, JB (2017). From Planar Cell Polarity to Ciliogenesis and Back: The Curious Tale of the PPE and CPLANE proteins. Trends Cell Biol 27, 379-390.

Al Jord, A, Shihavuddin, A, Servignat d'Aout, R, Faucourt, M, Genovesio, A, Karaiskou, A, SobczakThépot, J, Spassky, N, and Meunier, A (2017). Calibrated mitotic oscillator drives motile ciliogenesis. Science 358, 803-806.

Amsterdam, A, Nissen, RM, Sun, Z, Swindell, EC, Farrington, S, and Hopkins, N (2004). Identification of 315 genes essential for early zebrafish development. Proc Natl Acad Sci USA 101, 12792-12797.

Badano, JL, Leitch, CC, Ansley, SJ, May-Simera, H, Lawson, S, Lewis, RA, Beales, PL, Dietz, HC, Fisher, $\mathrm{S}$, and Katsanis, N (2006a). Dissection of epistasis in oligogenic Bardet-Biedl syndrome. Nature 439, 326-330.

Badano, JL, Mitsuma, N, Beales, PL, and Katsanis, N (2006b). The ciliopathies: an emerging class of human genetic disorders. Annu Rev Genomics Hum Genet 7, 125-148.

Bangs, F, and Anderson, KV (2017). Primary Cilia and Mammalian Hedgehog Signaling. Cold Spring Harb Perspect Biol 9.

BARNES, BG (1961). Ciliated secretory cells in the pars distalis of the mouse hypophysis. J Ultrastruct Res 5, 453-467.

Barr, MM, and Sternberg, PW (1999). A polycystic kidney-disease gene homologue required for male mating behaviour in C. elegans. Nature 401, 386-389.

Bernabé-Rubio, M, and Alonso, MA (2017). Routes and machinery of primary cilium biogenesis. Cell Mol Life Sci 74, 4077-4095.

Bernstein, M, Beech, PL, Katz, SG, and Rosenbaum, JL (1994). A new kinesin-like protein (Klp1) localized to a single microtubule of the Chlamydomonas flagellum. J Cell Biol 125, 1313-1326.

Birtel, J et al. (2017). Novel Insights Into the Phenotypical Spectrum of KIF11-Associated Retinopathy, Including a New Form of Retinal Ciliopathy. Invest Ophthalmol Vis Sci 58, 3950-3959.

Braun, DA, and Hildebrandt, F (2017). Ciliopathies. Cold Spring Harb Perspect Biol 9.

Broix, L et al. (2018). Ciliogenesis and cell cycle alterations contribute to KIF2A-related malformations of cortical development. Hum Mol Genet 27, 224-238.

Buchan, JG, Gray, RS, Gansner, JM, Alvarado, DM, Burgert, L, Gitlin, JD, Gurnett, CA, and Goldsmith, MI (2014). Kinesin family member 6 (kif6) is necessary for spine development in zebrafish. Dev Dyn 243, 1646-1657.

Cantaut-Belarif, Y, Sternberg, JR, Thouvenin, O, Wyart, C, and Bardet, P-L (2018). The Reissner Fiber in the Cerebrospinal Fluid Controls Morphogenesis of the Body Axis. Curr Biol. 
Cardenas-Rodriguez, M, Irigoín, F, Osborn, DPS, Gascue, C, Katsanis, N, Beales, PL, and Badano, JL (2013). The Bardet-Biedl syndrome-related protein CCDC28B modulates mTORC2 function and interacts with SIN1 to control cilia length independently of the mTOR complex. Hum Mol Genet 22, 4031-4042.

Carleton, M, Mao, M, Biery, M, Warrener, P, Kim, S, Buser, C, Marshall, CG, Fernandes, C, Annis, J, and Linsley, PS (2006). RNA interference-mediated silencing of mitotic kinesin KIF14 disrupts cell cycle progression and induces cytokinesis failure. Mol Cell Biol 26, 3853-3863.

Castillo, A, and Justice, MJ (2007). The kinesin related motor protein, Eg5, is essential for maintenance of pre-implantation embryogenesis. Biochem Biophys Res Commun 357, 694-699.

Chauvière, M, Kress, C, and Kress, M (2008). Disruption of the mitotic kinesin Eg5 gene (Knsl1) results in early embryonic lethality. Biochem Biophys Res Commun 372, 513-519.

Chauvin, T, Xie, F, Liu, T, Nicora, CD, Yang, F, Camp, DG, Smith, RD, and Roberts, KP (2012). A Systematic Analysis of a Deep Mouse Epididymal Sperm Proteome. Biol Reprod 87.

Chen, M-H, Gao, N, Kawakami, T, and Chuang, P-T (2005). Mice deficient in the fused homolog do not exhibit phenotypes indicative of perturbed hedgehog signaling during embryonic development. Mol Cell Biol 25, 7042-7053.

Cheung, HO-L, Zhang, X, Ribeiro, A, Mo, R, Makino, S, Puviindran, V, Law, KKL, Briscoe, J, and Hui, C-C (2009). The kinesin protein Kif7 is a critical regulator of Gli transcription factors in mammalian hedgehog signaling. Sci Signal 2, ra29.

Choksi, SP, Babu, D, Lau, D, Yu, X, and Roy, S (2014). Systematic discovery of novel ciliary genes through functional genomics in the zebrafish. Development 141, 3410-3419.

Cornfine, S, Himmel, M, Kopp, P, El Azzouzi, K, Wiesner, C, Krüger, M, Rudel, T, and Linder, S (2011). The kinesin KIF9 and reggie/flotillin proteins regulate matrix degradation by macrophage podosomes. Mol Biol Cell 22, 202-215.

Cross, RA, and McAinsh, A (2014). Prime movers: the mechanochemistry of mitotic kinesins. Nat Rev Mol Cell Biol 15, 257-271.

Dafinger, C et al. (2011). Mutations in KIF7 link Joubert syndrome with Sonic Hedgehog signaling and microtubule dynamics. J Clin Invest 121, 2662-2667.

DE ROBERTIS, E (1956). Morphogenesis of the retinal rods; an electron microscope study. J Biophys Biochem Cytol 2, 209-218.

Demonchy, R, Blisnick, T, Deprez, C, Toutirais, G, Loussert, C, Marande, W, Grellier, P, Bastin, P, and Kohl, L (2009). Kinesin 9 family members perform separate functions in the trypanosome flagellum. J Cell Biol 187, 615-622.

Endoh-Yamagami, S et al. (2009). The mammalian Cos2 homolog Kif7 plays an essential role in modulating Hh signal transduction during development. Curr Biol 19, 1320-1326. 
Falk, N, Lösl, M, Schröder, N, and Gießl, A (2015). Specialized Cilia in Mammalian Sensory Systems. Cells 4, 500-519.

Filges, I et al. (2013). Exome sequencing identifies mutations in KIF14 as a novel cause of an autosomal recessive lethal fetal ciliopathy phenotype. Clin Genet.

Fujikura, K, Setsu, T, Tanigaki, K, Abe, T, Kiyonari, H, Terashima, T, and Sakisaka, T (2013). Kif14 mutation causes severe brain malformation and hypomyelination. PLoS ONE 8, e53490.

Gache, V, Gomes, ER, and Cadot, B (2017). Microtubule motors involved in nuclear movement during skeletal muscle differentiation. Mol Biol Cell 28, 865-874.

Ghossoub, R, Molla-Herman, A, Bastin, P, and Benmerah, A (2011). The ciliary pocket: a onceforgotten membrane domain at the base of cilia. Biol Cell 103, 131-144.

Grimes, DT, Boswell, CW, Morante, NFC, Henkelman, RM, Burdine, RD, and Ciruna, B (2016). Zebrafish models of idiopathic scoliosis link cerebrospinal fluid flow defects to spine curvature. Science 352, 1341-1344.

Gruneberg, U, Neef, R, Li, X, Chan, EHY, Chalamalasetty, RB, Nigg, EA, and Barr, FA (2006). KIF14 and citron kinase act together to promote efficient cytokinesis. J Cell Biol 172, 363-372.

Guemez-Gamboa, A, Coufal, NG, and Gleeson, JG (2014). Primary cilia in the developing and mature brain. Neuron 82, 511-521.

Hameed, A, Bennett, E, Ciani, B, Hoebers, LPC, Milner, R, Lawrie, A, Francis, SE, and Grierson, AJ (2013). No evidence for cardiac dysfunction in Kif6 mutant mice. PLoS ONE 8, e54636.

He, M, Subramanian, R, Bangs, F, Omelchenko, T, Liem, KF, Kapoor, TM, and Anderson, KV (2014). The kinesin-4 protein Kif7 regulates mammalian Hedgehog signalling by organizing the cilium tip compartment. Nat Cell Biol 16, 663-672.

Heck, MM, Pereira, A, Pesavento, P, Yannoni, Y, Spradling, AC, and Goldstein, LS (1993). The kinesinlike protein KLP61F is essential for mitosis in Drosophila. J Cell Biol 123, 665-679.

Heidet, L et al. (2017). Targeted Exome Sequencing Identifies PBX1 as Involved in Monogenic Congenital Anomalies of the Kidney and Urinary Tract. J Am Soc Nephrol 28, 2901-2914.

Hirokawa, N, Niwa, S, and Tanaka, Y (2010). Molecular motors in neurons: transport mechanisms and roles in brain function, development, and disease. Neuron 68, 610-638.

Hirokawa, N, Noda, Y, Tanaka, Y, and Niwa, S (2009). Kinesin superfamily motor proteins and intracellular transport. Nat Rev Mol Cell Biol 10, 682-696.

Hoff, S et al. (2013). ANKS6 is a central component of a nephronophthisis module linking NEK8 to INVS and NPHP3. Nat Genet 45, 951-956.

Homma, N, Takei, Y, Tanaka, Y, Nakata, T, Terada, S, Kikkawa, M, Noda, Y, and Hirokawa, N (2003). Kinesin superfamily protein 2A (KIF2A) functions in suppression of collateral branch extension. Cell 114, 229-239. 
Hu, Z, Liang, Y, Meng, D, Wang, L, and Pan, J (2015). Microtubule-depolymerizing kinesins in the regulation of assembly, disassembly, and length of cilia and flagella. Int Rev Cell Mol Biol 317, 241265.

Hua, K, and Ferland, RJ (2018). Primary cilia proteins: ciliary and extraciliary sites and functions. Cell Mol Life Sci 75, 1521-1540.

Huber, C, and Cormier-Daire, V (2012). Ciliary disorder of the skeleton. Am J Med Genet C Semin Med Genet 160C, 165-174.

Insinna, C, Pathak, N, Perkins, B, Drummond, I, and Besharse, JC (2008). The homodimeric kinesin, Kif17, is essential for vertebrate photoreceptor sensory outer segment development. Dev Biol 316, 160-170.

Ishikawa, H, and Marshall, WF (2011). Ciliogenesis: building the cell's antenna. Nat Rev Mol Cell Biol 12, 222-234.

Jacquet, BV, Salinas-Mondragon, R, Liang, H, Therit, B, Buie, JD, Dykstra, M, Campbell, K, Ostrowski, LE, Brody, SL, and Ghashghaei, HT (2009). FoxJ1-dependent gene expression is required for differentiation of radial glia into ependymal cells and a subset of astrocytes in the postnatal brain. Development 136, 4021-4031.

Jenkins, PM, Hurd, TW, Zhang, L, McEwen, DP, Brown, RL, Margolis, B, Verhey, KJ, and Martens, JR (2006). Ciliary targeting of olfactory CNG channels requires the CNGB1b subunit and the kinesin-2 motor protein, KIF17. Curr Biol 16, 1211-1216.

Jiang, L, Tam, BM, Ying, G, Wu, S, Hauswirth, WW, Frederick, JM, Moritz, OL, and Baehr, W (2015). Kinesin family 17 (osmotic avoidance abnormal-3) is dispensable for photoreceptor morphology and function. FASEB J 29, 4866-4880.

Johnson, $\mathrm{K}$ et al. (2014). Kif11 dependent cell cycle progression in radial glial cells is required for proper neurogenesis in the zebrafish neural tube. Dev Biol 387, 73-92.

Kanai, Y, Wang, D, and Hirokawa, N (2014). KIF13B enhances the endocytosis of LRP1 by recruiting LRP1 to caveolae. J Cell Biol 204, 395-408.

Kim, S, Lee, K, Choi, J-H, Ringstad, N, and Dynlacht, BD (2015). Nek2 activation of Kif24 ensures cilium disassembly during the cell cycle. Nat Commun 6, 8087.

Kobayashi, T, Tsang, WY, Li, J, Lane, W, and Dynlacht, BD (2011). Centriolar kinesin Kif24 interacts with CP110 to remodel microtubules and regulate ciliogenesis. Cell 145, 914-925.

Konjikusic, MJ et al. (2018). Mutations in Kinesin family member 6 reveal specific role in ependymal cell ciliogenesis and human neurological development. PLoS Genet 14, e1007817.

Konno, A, Shiba, K, Cai, C, and Inaba, K (2015). Branchial Cilia and Sperm Flagella Recruit Distinct Axonemal Components. PLoS One 10, e0126005

Kozminski, KG, Beech, PL, and Rosenbaum, JL (1995). The Chlamydomonas kinesin-like protein FLA10 is involved in motility associated with the flagellar membrane. J Cell Biol 131, 1517-1527. 
Kraus, MR-C, Clauin, S, Pfister, Y, Di Maïo, M, Ulinski, T, Constam, D, Bellanné-Chantelot, C, and Grapin-Botton, A (2012). Two mutations in human BICC1 resulting in Wnt pathway hyperactivity associated with cystic renal dysplasia. Hum Mutat 33, 86-90.

Leaf, A, and Von Zastrow, M (2015). Dopamine receptors reveal an essential role of IFT-B, KIF17, and Rab23 in delivering specific receptors to primary cilia. Elife 4.

Lechtreck, KF (2015). IFT-Cargo Interactions and Protein Transport in Cilia. Trends Biochem Sci 40, 765-778.

Lechtreck, K-F, and Witman, GB (2007). Chlamydomonas reinhardtii hydin is a central pair protein required for flagellar motility. J Cell Biol 176, 473-482.

Lee, S-H, Joo, K, Jung, EJ, Hong, H, Seo, J, and Kim, J (2018). Export of membrane proteins from the Golgi complex to the primary cilium requires the kinesin motor, KIFC1. FASEB J 32, 957-968.

Lewis, TR, Kundinger, SR, Link, BA, Insinna, C, and Besharse, JC (2018). Kif17 phosphorylation regulates photoreceptor outer segment turnover. BMC Cell Biol 19, 25.

Lewis, TR, Kundinger, SR, Pavlovich, AL, Bostrom, JR, Link, BA, and Besharse, JC (2017). Cos2/Kif7 and Osm-3/Kif17 regulate onset of outer segment development in zebrafish photoreceptors through distinct mechanisms. Dev Biol 425, 176-190.

Liem, KF, He, M, Ocbina, PJR, and Anderson, KV (2009). Mouse Kif7/Costal2 is a cilia-associated protein that regulates Sonic hedgehog signaling. Proc Natl Acad Sci USA 106, 13377-13382.

Makrythanasis, P et al. (2018). Biallelic variants in KIF14 cause intellectual disability with microcephaly. Eur J Hum Genet 26, 330-339.

Mazelova, J, Astuto-Gribble, L, Inoue, H, Tam, BM, Schonteich, E, Prekeris, R, Moritz, OL, Randazzo, PA, and Deretic, D (2009). Ciliary targeting motif VxPx directs assembly of a trafficking module through Arf4. EMBO J 28, 183-192.

McHugh, T, Gluszek, AA, and Welburn, JPI (2018). Microtubule end tethering of a processive kinesin8 motor Kif18b is required for spindle positioning. J Cell Biol 217, 2403-2416.

Meunier, A, and Azimzadeh, J (2016). Multiciliated Cells in Animals. Cold Spring Harb Perspect Biol 8.

Mitchison, HM, and Valente, EM (2017). Motile and non-motile cilia in human pathology: from function to phenotypes. J Pathol 241, 294-309.

Miyamoto, T, Hosoba, K, Ochiai, H, Royba, E, Izumi, H, Sakuma, T, Yamamoto, T, Dynlacht, BD, and Matsuura, S (2015). The Microtubule-Depolymerizing Activity of a Mitotic Kinesin Protein KIF2A Drives Primary Cilia Disassembly Coupled with Cell Proliferation. Cell Rep.

Moawia, A et al. (2017). Mutations of KIF14 cause primary microcephaly by impairing cytokinesis. Ann Neurol 82, 562-577.

Morthorst, SK, Christensen, ST, and Pedersen, LB (2018). Regulation of ciliary membrane protein trafficking and signalling by kinesin motor proteins. FEBS J. 
Nachury, MV (2018). The molecular machines that traffic signaling receptors into and out of cilia. Curr Opin Cell Biol 51, 124-131.

Nakayama, K, and Katoh, Y (2018). Ciliary protein trafficking mediated by IFT and BBSome complexes with the aid of kinesin-2 and dynein-2 motors. J Biochem 163, 155-164.

Nath, S, Bananis, E, Sarkar, S, Stockert, RJ, Sperry, AO, Murray, JW, and Wolkoff, AW (2007). Kif5B and Kifc1 interact and are required for motility and fission of early endocytic vesicles in mouse liver. Mol Biol Cell 18, 1839-1849.

Niwa, S, Nakajima, K, Miki, H, Minato, Y, Wang, D, and Hirokawa, N (2012). KIF19A is a microtubuledepolymerizing kinesin for ciliary length control. Dev Cell 23, 1167-1175.

Novas, R et al. (2018). Kinesin 1 regulates cilia length through an interaction with the Bardet-Biedl syndrome related protein CCDC28B. Sci Rep 8, 3019.

Nozawa, YI, Yao, E, Lin, C, Yang, J-H, Wilson, CW, Gacayan, R, and Chuang, P-T (2013). Fused (Stk36) is a ciliary protein required for central pair assembly and motile cilia orientation in the mammalian oviduct. Dev Dyn 242, 1307-1319.

Olbrich, H et al. (2012). Recessive HYDIN mutations cause primary ciliary dyskinesia without randomization of left-right body asymmetry. Am J Hum Genet 91, 672-684.

Ostergaard, P et al. (2012). Mutations in KIF11 cause autosomal-dominant microcephaly variably associated with congenital lymphedema and chorioretinopathy. Am J Hum Genet 90, 356-362.

Park, TJ, Mitchell, BJ, Abitua, PB, Kintner, C, and Wallingford, JB (2008). Dishevelled controls apical docking and planar polarization of basal bodies in ciliated epithelial cells. Nat Genet 40, 871-879.

Pazour, GJ, Dickert, BL, Vucica, Y, Seeley, ES, Rosenbaum, JL, Witman, GB, and Cole, DG (2000). Chlamydomonas IFT88 and its mouse homologue, polycystic kidney disease gene tg737, are required for assembly of cilia and flagella. J Cell Biol 151, 709-718.

Peden, EM, and Barr, MM (2005). The KLP-6 kinesin is required for male mating behaviors and polycystin localization in Caenorhabditis elegans. Curr Biol 15, 394-404.

Pedersen, LB, Mogensen, JB, and Christensen, ST (2016). Endocytic Control of Cellular Signaling at the Primary Cilium. Trends Biochem Sci 41, 784-797.

Poirier, $\mathrm{K}$ et al. (2013). Mutations in TUBG1, DYNC1H1, KIF5C and KIF2A cause malformations of cortical development and microcephaly. Nat Genet 45, 639-647.

Putoux, A et al. (2011). KIF7 mutations cause fetal hydrolethalus and acrocallosal syndromes. Nat Genet 43, 601-606.

Putoux, A et al. (2012). Novel KIF7 mutations extend the phenotypic spectrum of acrocallosal syndrome. J Med Genet 49, 713-720.

Rahman, A, Kamal, A, Roberts, EA, and Goldstein, LS (1999). Defective kinesin heavy chain behavior in mouse kinesin light chain mutants. J Cell Biol 146, 1277-1288. 
Reilly, ML et al. (2018). Loss of function mutations in KIF14 cause severe microcephaly and kidney development defects in humans and zebrafish. Hum Mol Genet.

Reiter, JF, and Leroux, MR (2017). Genes and molecular pathways underpinning ciliopathies. Nat Rev Mol Cell Biol 18, 533-547.

Rink, JC, Gurley, KA, Elliott, SA, and Sánchez Alvarado, A (2009). Planarian Hh signaling regulates regeneration polarity and links $\mathrm{Hh}$ pathway evolution to cilia. Science 326, 1406-1410.

Ross, AJ, Dailey, LA, Brighton, LE, and Devlin, RB (2007). Transcriptional profiling of mucociliary differentiation in human airway epithelial cells. Am J Respir Cell Mol Biol 37, 169-185.

Sánchez, I, and Dynlacht, BD (2016). Cilium assembly and disassembly. Nat Cell Biol 18, 711-717.

Santos-Ledo, A, Garcia-Macia, M, Campbell, PD, Gronska, M, and Marlow, FL (2017). Kinesin-1 promotes chondrocyte maintenance during skeletal morphogenesis. PLoS Genet 13, e1006918.

Satir, P, and Christensen, ST (2007). Overview of structure and function of mammalian cilia. Annu Rev Physiol 69, 377-400.

Sawin, KE, LeGuellec, K, Philippe, M, and Mitchison, TJ (1992). Mitotic spindle organization by a plusend-directed microtubule motor. Nature 359, 540-543.

Scholey, JM (2013). Kinesin-2: a family of heterotrimeric and homodimeric motors with diverse intracellular transport functions. Annu Rev Cell Dev Biol 29, 443-469.

Schou, KB et al. (2017). KIF13B establishes a CAV1-enriched microdomain at the ciliary transition zone to promote Sonic hedgehog signalling. Nat Commun 8, 14177.

Schrøder, JM et al. (2011). EB1 and EB3 promote cilia biogenesis by several centrosome-related mechanisms. J Cell Sci 124, 2539-2551.

Shamseldin, HE, Yakulov, TA, Hashem, A, Walz, G, and Alkuraya, FS (2016). ANKS3 is mutated in a family with autosomal recessive laterality defect. Hum Genet.

Snow, JJ, Ou, G, Gunnarson, AL, Walker, MRS, Zhou, HM, Brust-Mascher, I, and Scholey, JM (2004). Two anterograde intraflagellar transport motors cooperate to build sensory cilia on $\mathrm{C}$. elegans neurons. Nat Cell Biol 6, 1109-1113.

Song, $\mathrm{R}$ et al. (2014). miR-34/449 miRNAs are required for motile ciliogenesis by repressing cp110. Nature 510, 115-120.

Song, Z, Zhang, X, Jia, S, Yelick, PC, and Zhao, C (2016). Zebrafish as a Model for Human Ciliopathies. J Genet Genomics 43, 107-120.

Sorokin, SP (1968). Reconstructions of centriole formation and ciliogenesis in mammalian lungs. J Cell Sci 3, 207-230.

Stout, JR, Yount, AL, Powers, JA, Leblanc, C, Ems-McClung, SC, and Walczak, CE (2011). Kif18B interacts with EB1 and controls astral microtubule length during mitosis. Mol Biol Cell 22, 3070-3080. 
Tanaka, K, Sugiura, Y, Ichishita, R, Mihara, K, and Oka, T (2011). KLP6: a newly identified kinesin that regulates the morphology and transport of mitochondria in neuronal cells. J Cell Sci 124, 2457-2465.

Tanaka, Y, Kanai, Y, Okada, Y, Nonaka, S, Takeda, S, Harada, A, and Hirokawa, N (1998). Targeted disruption of mouse conventional kinesin heavy chain, kif5B, results in abnormal perinuclear clustering of mitochondria. Cell 93, 1147-1158.

Tanenbaum, ME, Macurek, L, van der Vaart, B, Galli, M, Akhmanova, A, and Medema, RH (2011). A complex of Kif18b and MCAK promotes microtubule depolymerization and is negatively regulated by Aurora kinases. Curr Biol 21, 1356-1365.

Thomas, J, Morlé, L, Soulavie, F, Laurençon, A, Sagnol, S, and Durand, B (2010). Transcriptional control of genes involved in ciliogenesis: a first step in making cilia. Biol Cell 102, 499-513.

Verhey, KJ, and Hammond, JW (2009). Traffic control: regulation of kinesin motors. Nat Rev Mol Cell Biol 10, 765-777.

Vincensini, L, Blisnick, T, and Bastin, P (2011). 1001 model organisms to study cilia and flagella. Biol Cell 103, 109-130.

Vogel, P, Read, RW, Hansen, GM, Payne, BJ, Small, D, Sands, AT, and Zambrowicz, BP (2012). Congenital hydrocephalus in genetically engineered mice. Vet Pathol 49, 166-181.

Wakana, Y, Villeneuve, J, van Galen, J, Cruz-Garcia, D, Tagaya, M, and Malhotra, V (2013). Kinesin$5 / \mathrm{Eg} 5$ is important for transport of CARTS from the trans-Golgi network to the cell surface. J Cell Biol 202, 241-250.

Walczak, CE, Gayek, S, and Ohi, R (2013). Microtubule-depolymerizing kinesins. Annu Rev Cell Dev Biol 29, 417-441.

Walentek, P, Quigley, IK, Sun, DI, Sajjan, UK, Kintner, C, and Harland, RM (2016). Ciliary transcription factors and miRNAs precisely regulate $\mathrm{Cp} 110$ levels required for ciliary adhesions and ciliogenesis. Elife 5.

Wang, D, Nitta, R, Morikawa, M, Yajima, H, Inoue, S, Shigematsu, H, Kikkawa, M, and Hirokawa, N (2016). Motility and microtubule depolymerization mechanisms of the Kinesin-8 motor, KIF19A. Elife 5.

Wang, J, Silva, M, Haas, LA, Morsci, NS, Nguyen, KCQ, Hall, DH, and Barr, MM (2014). C. elegans ciliated sensory neurons release extracellular vesicles that function in animal communication. Curr Biol 24, 519-525.

Wheway, G, Nazlamova, L, and Hancock, JT (2018). Signaling through the Primary Cilium. Front Cell Dev Biol 6, 8.

Williams, CL, McIntyre, JC, Norris, SR, Jenkins, PM, Zhang, L, Pei, Q, Verhey, K, and Martens, JR (2014). Direct evidence for BBSome-associated intraflagellar transport reveals distinct properties of native mammalian cilia. Nat Commun 5, 5813. 
Wilson, CW, Nguyen, CT, Chen, M-H, Yang, J-H, Gacayan, R, Huang, J, Chen, J-N, and Chuang, P-T (2009). Fused has evolved divergent roles in vertebrate Hedgehog signalling and motile ciliogenesis. Nature 459, 98-102.

Wu, C-T, Chen, $\mathrm{H}-\mathrm{Y}$, and Tang, TK (2018). Myosin-Va is required for preciliary vesicle transportation to the mother centriole during ciliogenesis. Nat Cell Biol 20, 175-185.

Xu, Y, Takeda, S, Nakata, T, Noda, Y, Tanaka, Y, and Hirokawa, N (2002). Role of KIFC3 motor protein in Golgi positioning and integration. J Cell Biol 158, 293-303.

Yadav, SP, Sharma, NK, Liu, C, Dong, L, Li, T, and Swaroop, A (2016). Centrosomal protein CP110 controls maturation of the mother centriole during cilia biogenesis. Development 143, 1491-1501.

Yang, Z, Roberts, EA, and Goldstein, LS (2001a). Functional analysis of mouse C-terminal kinesin motor KifC2. Mol Cell Biol 21, 2463-2466.

Yang, Z, Xia, C, Roberts, EA, Bush, K, Nigam, SK, and Goldstein, LS (2001b). Molecular cloning and functional analysis of mouse C-terminal kinesin motor KifC3. Mol Cell Biol 21, 765-770.

Yin, X, Takei, Y, Kido, MA, and Hirokawa, N (2011). Molecular motor KIF17 is fundamental for memory and learning via differential support of synaptic NR2A/2B levels. Neuron 70, 310-325.

Yokoyama, R, O'toole, E, Ghosh, S, and Mitchell, DR (2004). Regulation of flagellar dynein activity by a central pair kinesin. Proc Natl Acad Sci USA 101, 17398-17403.

Yu, K, McGlynn, S, and Matise, MP (2013). Floor plate-derived sonic hedgehog regulates glial and ependymal cell fates in the developing spinal cord. Development 140, 1594-1604.

Yue, Y, Blasius, TL, Zhang, S, Jariwala, S, Walker, B, Grant, BJ, Cochran, JC, and Verhey, KJ (2018). Altered chemomechanical coupling causes impaired motility of the kinesin-4 motors KIF27 and KIF7. J Cell Biol 217, 1319-1334.

Zhao, C, Omori, Y, Brodowska, K, Kovach, P, and Malicki, J (2012). Kinesin-2 family in vertebrate ciliogenesis. Proc Natl Acad Sci USA 109, 2388-2393. 


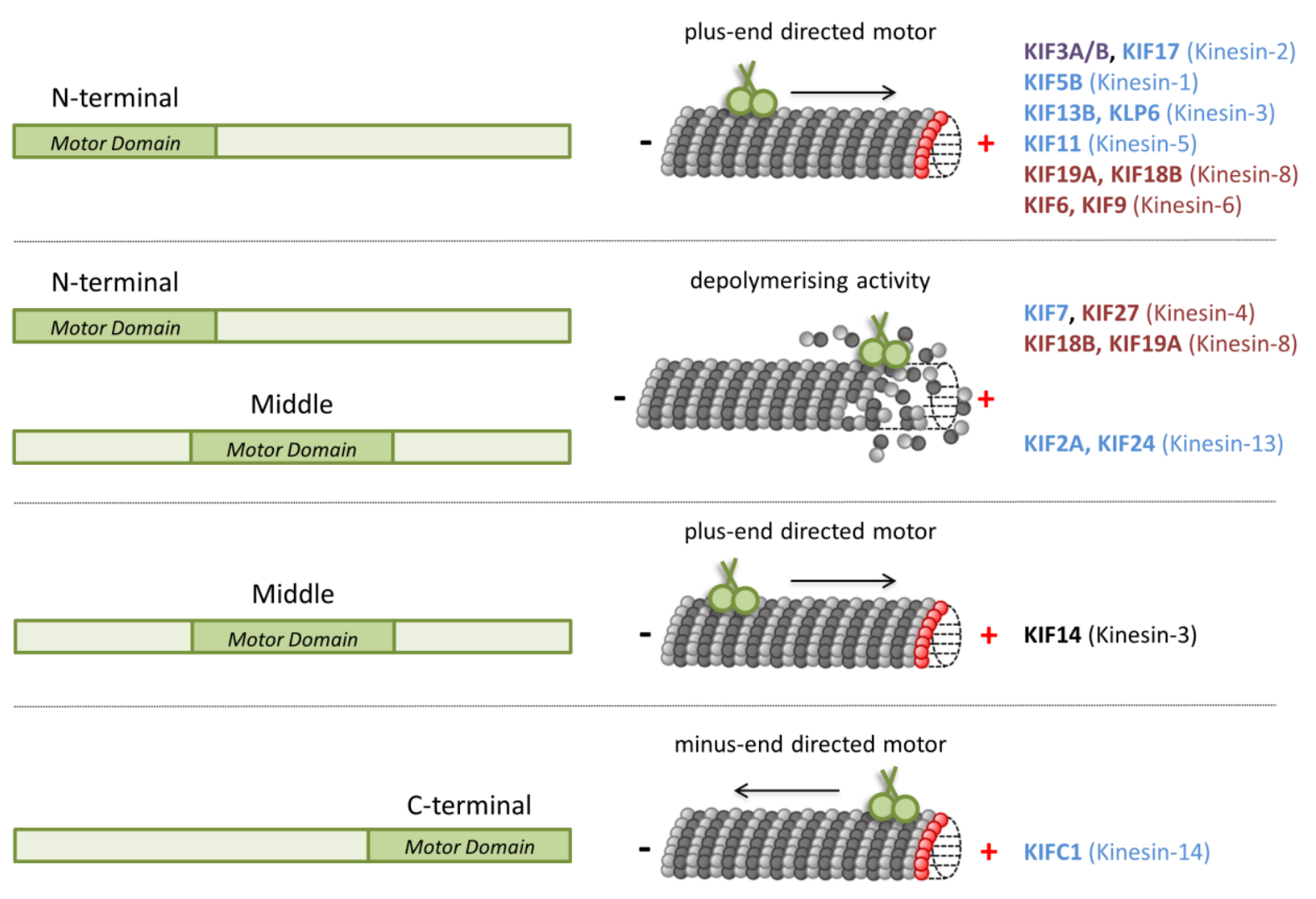

Figure 1: Functional Kinesin families.

Kinesin family members (KIFs) can be classified based on the localization of their motor domain ( $\mathrm{N}$ terminal, middle, C-terminal) and their activity (motor or depolymerizing). Kinesins involved in ciliary functions are indicated on the right depending on this classification. Kinesin involved in PC-specific functions are indicated in blue, those in motile cilia functions are indicated in red and those involved in both types of cilia in purple. 


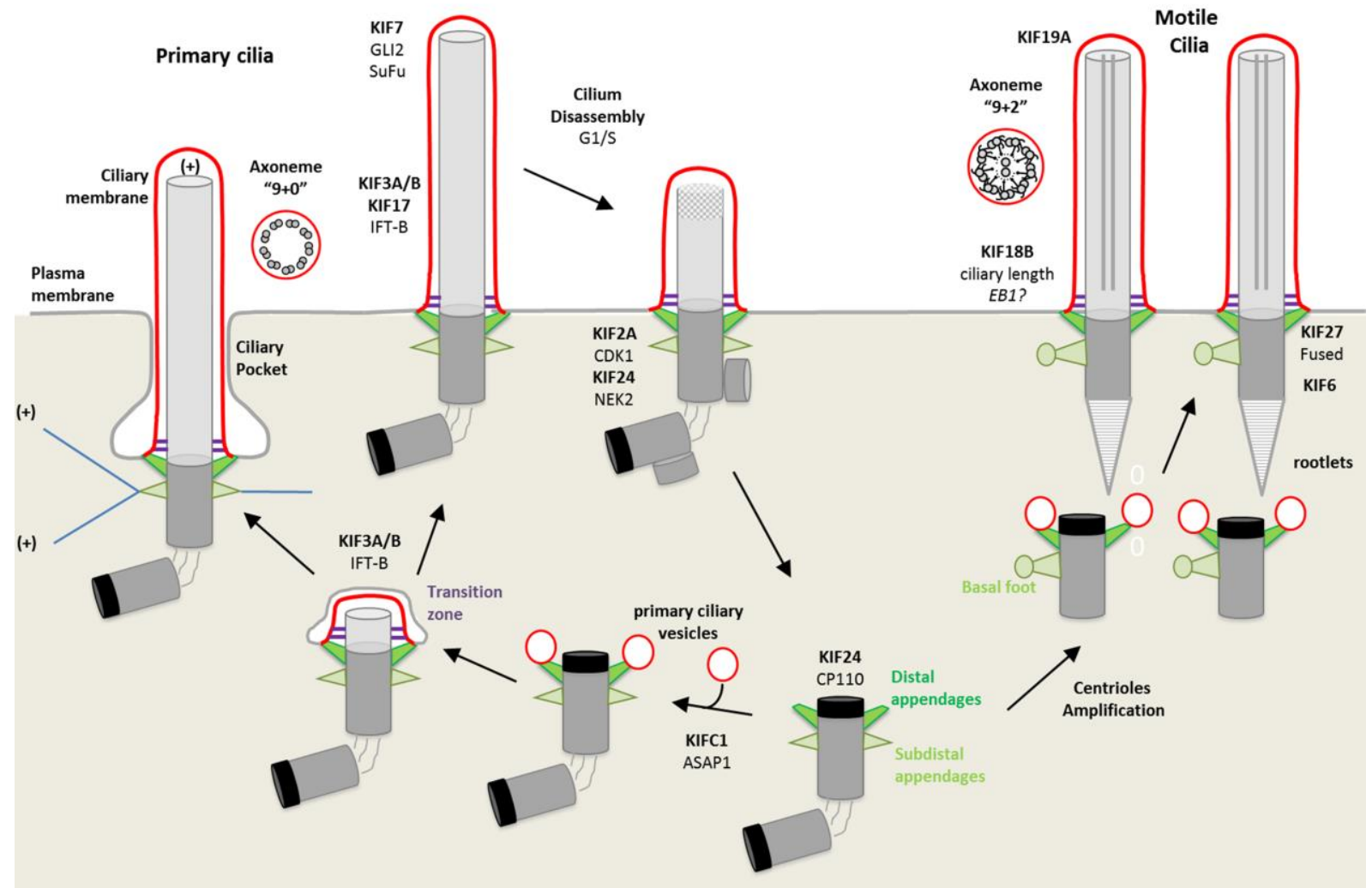

Figure 2: Role of kinesins at primary and motile cilia.

The scheme represents ciliogenesis pathways for primary (left) and motile (right) cilia. Ciliary KIFs are indicated in the scheme together with their known functional partners where they are known to act. 


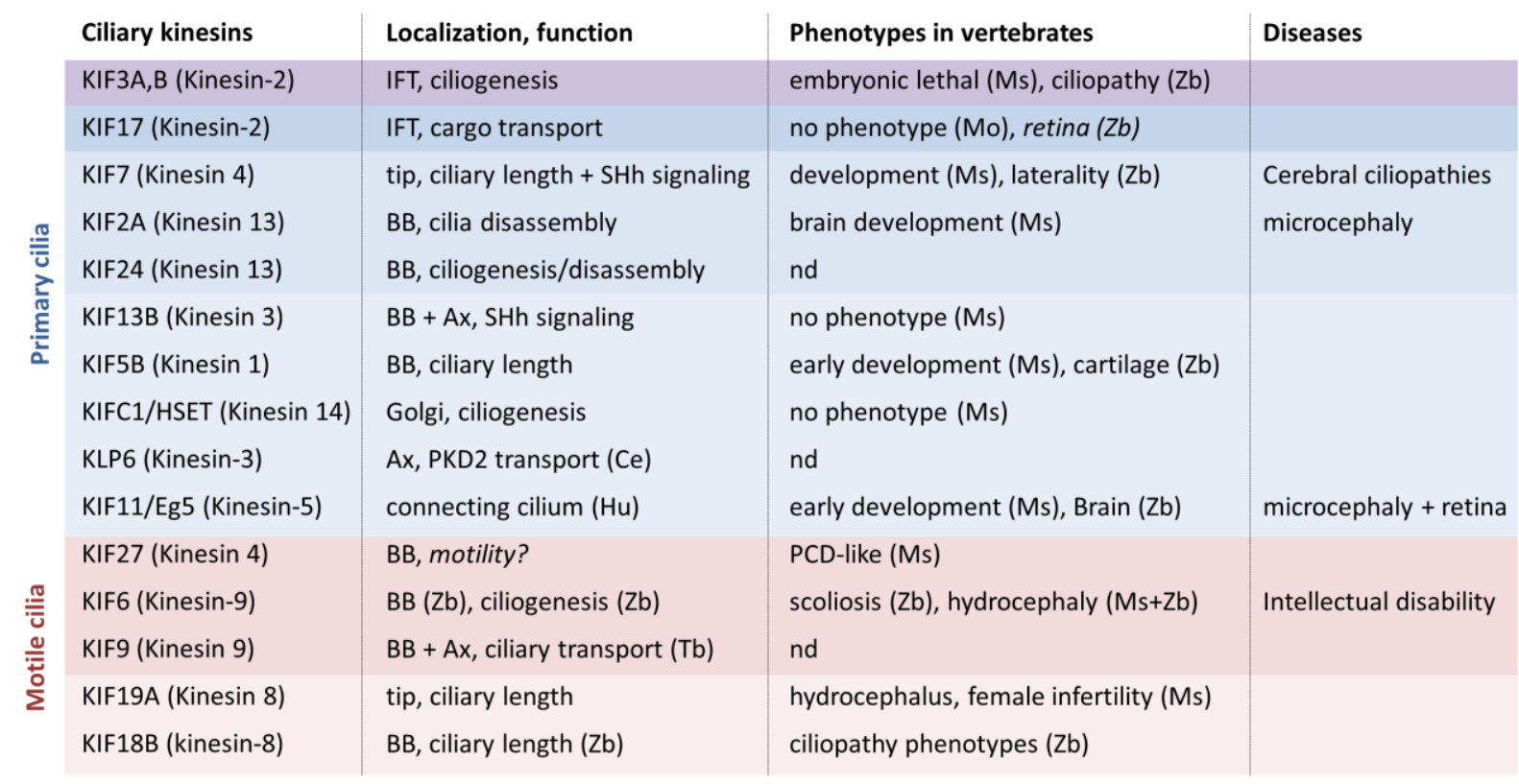

Table 1: Ciliary kinesins.

Localization, and in vitro and in vivo functions of ciliary kinesins are indicated in the table. Different colors are used to stress KIFs involved in either PC (blue), motile cilia (pink) or both (purple). Indicated in vivo phenotypes are for mouse models except when indicated. Zb: Zebrafish; Ce Caenorhabditis elegans; Tb: Trypanosoma bruceї; Ax: Axoneme; BB: Basal body; IFT: Intraflagellar transport; SHh: Sonic Hedgehog. 